\title{
Hepatic Lipoma: Radiological Imaging Findings
}

\author{
Mustafa Koplay ${ }^{1}$, Alper Hacioglu ${ }^{2}$, Mustafa Cem Algin ${ }^{2}$ \\ ${ }^{1}$ Department of Radiology, Selcuklu Medical Faculty, Selcuk University, Konya, Turkey \\ ${ }^{2}$ Department of General Surgery, Medical Faculty, Dumlupinar University, Kutahya, Turkey \\ E-mail: koplaymustafa@hotmail.com
}

Received November 26, 2010; revised March 21, 2011; accepted August 17, 2011

\begin{abstract}
Hemangiomas and hepatic metastases are the leading reasons of echogenic masses on ultrasound (US) evaluation of the liver. Lipomas of the liver are extremely rare and have been sporadically reported in the literature during the last century. The present report describes a patient with hepatic lipoma together with liver metastases from gastric adenocarcinoma. A 54 years old woman was refered to our department because of abdominal pain. Patient has been operated for gastric adenocarcinoma 3 months ago she was evaluated with US, computed tomography (CT) and magnetic resonance imaging (MRI). The abdominal US revealed a $12 \times 10 \mathrm{~mm}$ echogenic mass with smooth borders in 7th segment of the liver. CT scan showed a hypodense lesion in the same hepatic segment with fat dencity and no contrast involvement. MRI demonstrated the same lesion on T1 and T2 weighted images as hyperintence mass. The final radiographic diagnosis was hepatic lipoma. However, there was metastas in the liver of patient. Patient died 4 months later due to metastatic gastric adenocarcinoma. Hepatic lipoma should be kept in mind in echogenic masses on US evaluation of the liver.
\end{abstract}

Keywords: Lipoma, Liver, Different Diagnosis

\section{Introduction}

Although ultrasound (US) is the most used imaging modality in screaning of the focal liver lesions nowadays, the US may not characterize the nodules indwelled in liver and computed tomography (CT) scan, MRI and/or needle biopsy may be required for differential diagnosis [1]. Hemangiomas and hepatic metastases are the leading reasons of echogenic mass on ultrasonographic evaluation of the liver [2]. Hepatic lipomas appear as hyperechoic masses on ultrasonographic evaluation. The etiology of these lesions is not well defined and their clinical manifestations vary a lot, but often they are asymptomatic.

We report a case of patient with hepatic lipoma and discuss the radiographic findings. Also we discuss differential diagnosis of the hepatic lipoma.

\section{Case Report}

A 54 years old woman was refered to our department because of mass in upper-outer quadrant of the right breast and abdominal pain. Patient has been operated for gastric adenocarcinoma 3 months ago and has been sub- jected to total gastrectomy, esophagojejunostomy and Braun anastomosis. Patient have moderate to severe symptoms of bile reflux esophagitis and abdominal discomfort and ascites. Tru-cut biopsy was performed on breast mass and histopathologic examination was not able to make differential diagnosis between primary breast ductal carcinoma and metastatic gastric adenocarcinoma, but the primary breast tumor is eventual diagnosis. Quadrantectomy and axillar disection was planned, but due to comorbid problems the operation was suspended. The tumor staging examinations was performed.

The abdominal US revealed a $40 \times 30 \mathrm{~mm}$ hypoechoic mass in 5th segment and a $12 \times 10 \mathrm{~mm}$ echogenic mass in 7th segment of the liver [Figure 1]. It showed also abdominal ascites. Due to metastatic suspicion an abdominal CT scan was performed. CT scan demonstrated a metastasis compatible hypodense mass with periferal contrast involvement (50 HU) in 5th segment and hypodense mass at fat density $(-80 \mathrm{HU})$ with no contrast involvement in 7 th segment of the liver [Figure 2]. For futher evaluation an abdominal MRI was performed. T1 and $\mathrm{T} 2$ weighted images showed hyperintence lesion in the 7th segment of the liver [Figure 3]. The final radiological diagnosis was hepatic lipoma according to $\mathrm{CT}$ 


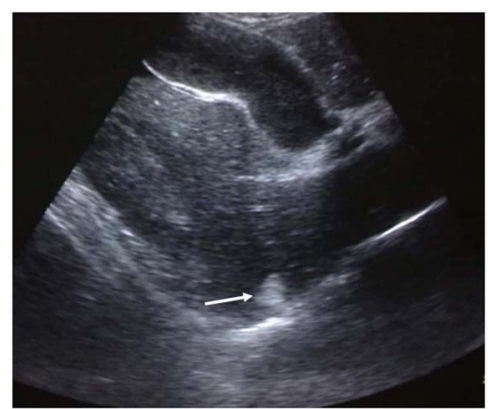

Figure 1. US image shows the echogenic lesion in 7th segment of the liver (arrow).

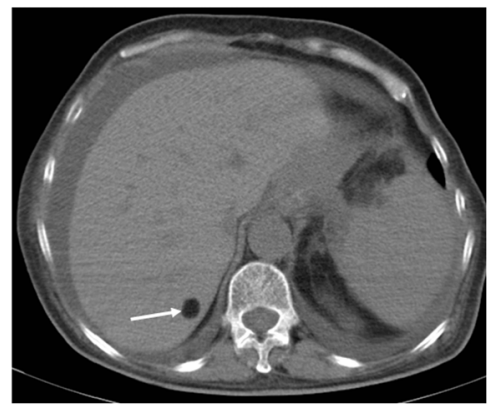

Figure 2. CT axial image shows the hypodense lesion at fat density $(-80 \mathrm{HU})$ in the liver (arrow) and perihepatic ascites.

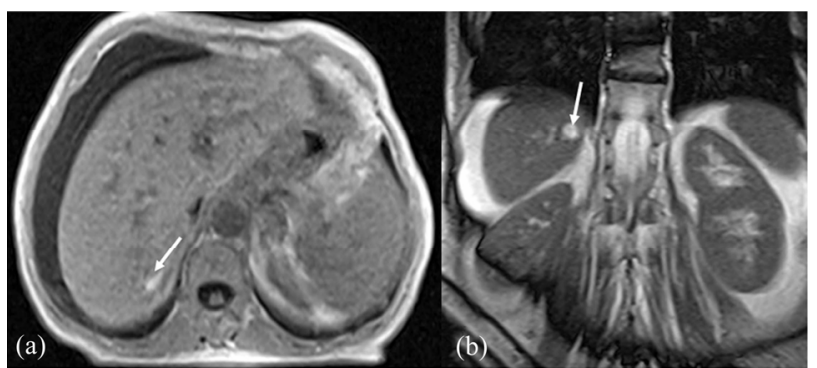

Figure 3. In MRI, T1 axial (a) and T2 coronal (b) weighted images shows hyperintence lesion in liver (arrows).

and MRI findings.

During these examinations the physical condition of the patient was gradually deteriorated and she died 4 months later.

\section{Discussion}

Hemangiomas and hepatic metastases are the leading reasons of echogenic masses on ultrasonographic evaluation of the liver. Hemangiomas are the most common benign hepatic tumors and usually are below $3 \mathrm{~cm}$ in diameter. Metastases are the most common malignant tumors of the liver and generally are hypoechogenic, but some gastrointestinal metastasis may appear echogenic. If so, they are multiple and have periferal hypo or anech- oic halo [2].

Hepatic lesions may contain macroscopic fat or intracellular lipids. Macroscopic fat containing lesions of the liver are angiomyolipoma, lipoma, liposarcoma, theratoma, pseudolipoma of the Glisson's capsule, inrahepatic pericaval fat, extramedullary hematopoiesis and metastases. Lesions containing intracellular lipids are focal steatosis, adenoma, focal nodular hyperplasia, regenerative nodules and hepatocellular carcinoma $[1,3]$.

Fat usually appears hyperechoic at US, although fat in some regions may appear hypoechoic [4]. Fat attenuates sound more than the adjacent liver parenchyma, so partial acoustic shadowing may occur deep to fatty tumors. Fat is of low attenuation (hypodense) compared with normal liver parenchyma at computed tomography (CT), with a range of -10 to $-100 \mathrm{HU}$, and high in signal intensity (hyperintence) on T1-weighted magnetic resonance (MR) images [5]. In addition, several MR imaging sequences aid in the detection of fat, including fat suppression sequences (hypointence) and chemical shift imaging with opposed-phase gradient-echo sequences $[6,7]$.

There are two masses with different imaging properties in the present case. One of them is compatible with gastric cancer metastasis and the second is compatible with hepatic lipoma, therefore both do not require surgery.

Although some hepatic lipomas may mimick angiomiolipomas $[8,9]$, they don't carry malignant potential and there are many characteristic findings on US, CT and MRI, which are satisfactory evidences for hepatic lipoma diagnosis. Therefore many authors do not recommend more aggressive diagnostic procedures, such as needle liver biopsy and operation [10]. We also believe that awareness from characteristic imaging findings of hepatic lipoma may prevent redundant invasive procedures.

\section{References}

[1] C. Basaran, M. Karcaaltincaba, D. Akata, N. Karabulut, D. Akinci, M. Ozmen and O. Akhan, "Fat-Containing Lesions of the Liver: Cross-Sectional Imaging Findings with Emphasis on MRI," American Journal of Roentgenology, Vol. 184, No. 4, 2005, pp. 1103-1110.

[2] C. C. Reading and J. W. Charboneau, "Case of the Day. Ultrasound. Hepatic Lipoma," Radiographics, Vol. 10, No. 3, 1990, pp. 511-512.

[3] S. R. Prasad, H. Wang, H. Rosas, C. O. Menias, V. R. Narra, W. D. Middleton and J. P. Heiken, "Fat-Containing Lesions of the Liver: Radiologic-Pathologic Correlation," Radiographics, Vol. 25, No. 2, 2005, pp. 321-331.

[4] G. Heinz-Peer, C. Oettl, G. Mayer and G. H. Mostbeck, "Hypoechoic Perirenal Fat in Renal Transplant Recipients," Radiology, Vol. 193, No. 3, 1994, pp. 717-720.

[5] F. Musante, L. E. Derchi, F. Zappasodi, M. Bazzocchi, G. 
C. Riviezzo, A. Banderali and G. R. Cicio, "Myelolipoma of the Adrenal Gland: Sonographic and CT Features," American Journal of Roentgenology, Vol. 151, No. 5, 1988, pp. 961-964.

[6] D. Mathieu, M. Paret, A. E. Mahfouz, F. Caseiro-Alves, J. Tran Van Nhieu, M. C. Anglade, et al., "Hyperintense Benign Liver Lesions on Spin-Echo T1-Weighted MR Images: Pathologic Correlations," Abdominal Imaging, Vol. 22, No. 4, 1997, pp. 410-417. doi: $10.1007 / \mathrm{s} 002619900222$

[7] E. M. Delfaut, J. Beltran, G. Johnson, J. Rousseau, X. Marchandise and A. Cotton, "Fat Suppression in MR Imaging: Techniques and Pitfalls," Radiographics, Vol. 19, No. 2, 1999, pp. 373-382. doi:10.1007/s00595-008-3921-5

[8] N. Nakamura, A. Kudo, K. Ito, S. Tanaka and S. Arii, “A Hepatic Lipoma Mimicking Angiomyolipoma of the Liver: Report of a Case," Surgery Today, Vol. 39, No. 9, 2009, pp. 825-828.

[9] A. Nonomura, Y. Mizukami, K. Shimizu, M. Kadoya and O. Matsui, "Angiomyolipoma Mimicking True Lipoma of the Liver: Report of Two Cases," Pathology International, Vol. 46, No. 3, 1996, pp. 221-227. doi:10.1111/j.1440-1827.1996.tb03602.x

[10] L. Martí-Bonmatí, F. Menor, I. Vizcaino and J. Vilar, "Lipoma of the Liver: US, CT, and MRI Appearance," Gastrointestinal Radiology, Vol. 14, No. 2, 1989, pp. 155-157. 